\title{
CHEMOENZYMATIC PREPARATION OF CHONDROITIN SULFATES WITH A DEFINED SULFATION PATTERN
}

\author{
Agatha Bastida ${ }^{\mathrm{a}}$, Ernesto Doncel-Pérez ${ }^{\mathrm{b}}$, Pilar Montero-Calle ${ }^{\mathrm{a}}$, Victor Moreno-Zafra ${ }^{\mathrm{a}}$, \\ Leoncio Garrido ${ }^{c}$, Julia Revuelta ${ }^{a}$, Alfonso Fernández-Mayoralas ${ }^{a}$ and Eduardo García- \\ Junceda $^{\mathrm{a}}$ \\ a Departamento de Química Bio-Orgánica, Instituto de Química Orgánica General, \\ CSIC. 28006 Madrid, Spain. \\ ${ }^{\mathrm{b}}$ Unidad Neurología Experimental, Hospital Nacional de Parapléjicos, SESCAM. \\ 45071 Toledo, Spain \\ c Departamento de Química Física, Instituto de Ciencia y Tecnología de Polímeros, \\ CSIC. 28006 Madrid, Spain.
}

It is known that the proteoglycans present in the extracellular matrix, promote growth and differentiation of neural cells during development and maturation of the central nervous system. These properties are primarily associated to the carbohydrate chain of the glycosaminoglycan; in particular, to polysaccharide chains of heparan and chondroitin sulfates (HC and CS). Therefore, the synthesis of functionalized polymers that mimic the essential features of the HS and CS from the extracellular matrix to promote differentiation and guide axonal growth of neural cells is of great interest in the treatment of central nervous system injuries. In recent years, it has been shown the importance of specific sulfation patterns of glycosaminoglycan to regulate neurite outgrowth and axon guidance. In fact, it has been proposed that the molecular diversity of sulfated GAG's could provide a "sulfate code" that regulates neural development [1].

Herein, in this communication we present a chemoenzymatic strategy to prepare CS with defined pattern of sulfation. In this approach, we start from a commercial CD of bovine origin having $25 \%$ of sulfate groups at position 6 of $\mathrm{N}$-acetylgalactosamine and $75 \%$ of sulfate groups at position 4 . The treatment of this material with a recombinant endosulfatasa from Bacteroides thetaiotaomicron enable the preparation of CS with defined ratios of sulfation at position 6 and 4 .

The capacity of the various CS obtained to promote the neuronal differentiation has been assayed in cultures of PC12 cells.

[1] Holt, C. E., Dickson, B. J., Sugar codes for axons? Neuron 46, 169-172, (2005). 